\title{
RESET
}

Recherches en sciences sociales sur Internet

\section{La légitimité du jazz et des musiques savantes}

Des statistiques sur les publics à la critique en ligne

The legitimacy of jazz and learned music, from statistics on audiences to online criticism

\section{Wenceslas Lizé}

\section{(QpenEdition \\ Journals}

Édition électronique

URL : http://journals.openedition.org/reset/622

DOI : $10.4000 /$ reset.622

ISSN : 2264-6221

Éditeur

Association Recherches en sciences sociales sur Internet

\section{Référence électronique}

Wenceslas Lizé, «La légitimité du jazz et des musiques savantes », RESET [En ligne], 5 | 2016, mis en ligne le 30 juin 2016, consulté le 30 avril 2019. URL : http://journals.openedition.org/reset/622 ; DOI $10.4000 /$ reset.622

Ce document a été généré automatiquement le 30 avril 2019.

(c) Association Recherches en sciences sociales sur Internet 


\title{
La légitimité du jazz et des musiques savantes
}

\author{
Des statistiques sur les publics à la critique en ligne \\ The legitimacy of jazz and learned music, from statistics on audiences to online \\ criticism
}

Wenceslas Lizé

\section{Introduction}

1 Focalisées sur l'influence de la critique culturelle sur les choix des consommateurs (Allen \& Lincoln, 2004 ; Béra, 2003 ; François, 2008 ; King, 2007 ; Schmutz, 2005 ; Shrum, 1991), les sciences sociales traitent rarement du pouvoir de prescription qu'elle exerce à un niveau plus profond et généralement moins perçu, celui des critères de jugement (van Rees, 1987), des catégories d'appréciation (Roueff, 2013) et de la définition des figures d'amateur. C'est ce dont il est question dans cet article ${ }^{1}$ qui s'intéresse dans une perspective comparative, à la critique en ligne spécialisée dans le jazz et dans les musiques dites savantes. On cherchera notamment à identifier les modes de désignation des amateurs. Les figures d'amateurs prescrites par la critique en ligne, pour ce qu'elles révèlent non seulement du rapport à la musique attendu dans ces genres musicaux, mais aussi pour interroger leur légitimité culturelle sous ce nouvel angle. Nous verrons, en effet, que ces figures traduisent la valeur sociale conférée à chaque genre artistique, au moins par ceux qui le commentent, et renvoient aux lecteurs un sentiment de légitimité ou d'illégitimité du goût correspondant. L'enjeu de l'article, dans le cadre de ce numéro, est de montrer comment une investigation conduite en ligne, sur des webzines, permet de prolonger l'analyse de processus sociaux plus larges comme celui de la légitimation culturelle.

2 La première partie de l'article s'attache à l'étude comparée de la légitimité culturelle des «musiques savantes » (opéra, musique classique, musique contemporaine) et du jazz à partir d'indicateurs classiques : la reconnaissance accordée par l'État, la place au sein des 
institutions d'enseignement, la reconnaissance par des instances de consécration généralistes et surtout les caractéristiques sociales de leur audience. Ce dernier indicateur s'appuie sur le traitement secondaire des enquêtes consacrées aux pratiques culturelles des Français (de 1973 à 2008) qui permet de développer la comparaison en tenant compte des variations dans une perspective diachronique. L'élitisme croissant du goût pour le jazz a partie liée avec l'esthétisation du répertoire et l'institutionnalisation de la création, de la diffusion et de l'enseignement de ce genre musical en France. Ainsi, le jazz a connu au cours des cinquante dernières années un processus de légitimation qui le rapproche des «musiques savantes» dans l'espace socialement hiérarchisé des goûts. Pour autant, peut-on considérer que le jazz est désormais aussi consacré socialement que les « musiques savantes»?

Les débats dont font l'objet les analyses en termes de hiérarchisation sociale des goûts et de légitimité culturelle invitent à diversifier les indicateurs pour appréhender notamment les manières de commenter, de fréquenter et d'apprécier les œuvres qui, en matière de goût et de distinction, comptent autant que les préférences exprimées (Bourdieu, 1979). La seconde partie s'intéressera ainsi aux prescriptions de la critique en ligne concernant le rapport au jazz et aux musiques savantes. Comment la presse spécialisée, prescriptrice de manières de fréquenter les genres culturellement légitimes (Schrum, 1991; Roueff, 2013), désigne-t-elle l'amateur - au sens de personne qui aime la musique - dans chacun des genres étudiés ? Quelles significations sont associées à chaque catégorie employée pour nommer l'amateur? Plus fondamentalement, quelles réponses peut-on apporter à la question de la légitimité des «musiques savantes » et du jazz si l'on déplace le regard des données statistiques analysées dans la première partie vers la critique en ligne? On tentera de répondre à ces questions à partir d'une recherche lexicographique sur deux magazines spécialisés diffusés sur Internet, citizenjazz.com et resmusica.com. Apparus au début de la décennie 2000, ces deux sites s'inscrivent moins dans l'appropriation de l'activité de critique par des amateurs (Pasquier et al., 2014) que dans celle d'Internet par des domaines traditionnels d'exercice de la critique pourtant très attachés à la presse. L'intérêt pour la critique s'inscrit également dans l'étude des médiations sociales et des intermédiaires qui interviennent dans la relation entre production et réception des biens symboliques, notamment dans l'établissement des correspondances que font apparaitre les statistiques entre des catégories de biens (ici des genres musicaux) et des catégories de consommateurs déterminées (Bourdieu, 1977 ; Lizé, 2010 ; Roueff, 2013). De ce point de vue, les webzines spécialisés présentent l'intérêt de compter parmi les nouveaux intermédiaires numériques apparus avec le développement d'Internet, tout en s'inscrivant, pour ce qui est du jazz et des musiques savantes, dans la continuité des discours proposés par la critique papier.

\section{Consécration du jazz et recomposition de la légitimité culturelle}

4 Entre la première enquête sur les pratiques culturelles des Français (1973) et celle conduite par le ministère de la Culture en 2008, de nombreuses mutations ont affecté le rapport à la musique et la structure des relations entre les répertoires et les groupes sociaux. Une série d'innovations technologiques (commercialisation de la chaîne hi-fi, de la platine laser et du baladeur, développement des technologies numériques et diffusion de la musique en ligne) ont favorisé la diversification et l'accessibilité des répertoires 
diffusés. Parallèlement, l'industrie du disque s'organise depuis la décennie 1970 comme un système de concurrence internationale souvent décrit comme un « oligopole à frange concurrentielle » (Curien \& Moreau, 2006). Associée à l'augmentation sans précédent du nombre de musiciens ${ }^{2}$, cette structuration a favorisé la prolifération des productions ainsi qu'une diversification des répertoires (nouveaux genres et styles musicaux), dont la valorisation et la diffusion ont été de plus en plus soutenues par des stratégies de conquête des publics (promotion, marketing). Cet effet d'offre a été largement accompagné par les politiques culturelles qui, à partir des années 1980, ont soutenu les industries culturelles, l'emploi des artistes et ont substitué, ou du moins associé, à l'objectif de la « démocratisation culturelle » celui de la « démocratie culturelle » qui vise à remettre en cause les hiérarchies culturelles (Dubois, 1999: 279) en reconnaissant des genres dits « populaires » comme, dans le domaine musical, le jazz, le rock, les musiques du monde ou encore le rap, regroupés au sein de la catégorie institutionnelle des « musiques actuelles».

5 Ces transformations ont amplement contribué à la progression spectaculaire de l'écoute musicale (Donnat, 2009) et ne sont pas étrangères aux modifications de la structure des relations entre les positions sociales et les goûts musicaux, notamment au déplacement du jazz au sein du goût cultivé. La diversification de l'offre musicale et des modes d'écoute figure parmi les principaux facteurs explicatifs de l'éclectisme croissant des préférences musicales constaté au sein des classes supérieures, qui domine la sociologie du goût depuis le milieu des années 1990 (Peterson \& Simkus, 1992; Donnat, 1994; Peterson \& Kern, 1996; Coulangeon, 2003). Bien que nombre de ces travaux aient voulu voir dans l'opposition « omnivore/univore » une alternative au modèle de la distinction (Bourdieu, 1979) et un affaiblissement des hiérarchies culturelles qui résulterait notamment d'un changement de comportement culturel et social, Philippe Coulangeon souligne que « les transformations de la cartographie sociale des goûts ne sont pas synonymes d'affaiblissement des différences culturelles entre les groupes sociaux» (2010: 94). Plus fondamentalement, l'hypothèse de la montée de l'éclectisme souffre de la rareté des travaux susceptibles d'en administrer la preuve par une analyse longitudinale (Warde et al., 2007) et des réserves interprétatives émises dans ce genre d'entreprise (Coulangeon, 2010) en raison notamment de l'introduction de nouveaux genres au sein des nomenclatures. Peterson (2005) a lui-même montré que ses prédictions de 1992 étaient contredites par la régression de l'omnivorité au sein des classes supérieures dans les résultats de l'enquête états-unienne de 2002. Les sociologues sont de plus en plus nombreux à émettre des réserves sur les plans méthodologiques et analytiques des travaux sur l'omnivorisme culturel (Warde et al., 2007; Savage \& Gayo, 2011) et sur l'ampleur voire l'existence même de l'éclectisme au sein des classes supérieures (Prieur et al., 2008 ; Atkinson, 2011 ; Robette \& Roueff, 2014). Ainsi, l'omnivorisme apparait à Olivier Roueff et Nicolas Robette "comme un phénomène au mieux secondaire, au pire marginal » selon la façon dont on le mesure (2014 : 37). Toutefois, l'éclectisme demeure une modalité intéressante des configurations gustatives. Sans doute contribue-t-elle, au moins comme norme effectivement présente dans les principaux medias de la culture cultivée (Télérama, France Culture, Arte), à la recomposition de la légitimité culturelle. Mais l'ascension du jazz, par exemple, apparaît, dans le domaine musical, comme un mouvement plus tangible de cette recomposition, qui, du reste, peut contribuer à l'éclectisme des classes supérieures si on mesure ce dernier par le nombre de genres écoutés. 


\section{La légitimation du jazz : un cas d'école}

Parmi les oppositions canoniques entre producteurs et auditeurs, musiciens amateurs et musiciens professionnels, spectacle vivant et production discographique qui sous-tendent un monde musical très hétérogène, le clivage entre «savant » et " populaire » demeure sans doute l'une des plus structurantes (Coulangeon, 2004). Or, le cas du jazz met à mal cette opposition entre "musiques savante » ou " sérieuse » et " musique populaire » ou « actuelle » (variétés, rock, rap, etc.). S'il est vrai que du point de vue des formes d'emploi, de l'organisation du travail et de la définition du métier (interprète "exécutant » des musiques savantes/interprète "créateur " des musiques populaires), le jazz se situe plutôt du côté des musiques populaires (Coulangeon, 1999; Perrenoud, 2007), il occupe sur le marché du disque une position proche de celle de la «musique savante » par le volume global des ventes (moins de $4 \%$ ), la rareté des vedettes présentes au sein des hitparades et la moindre sensibilité à la crise que traverse le secteur depuis le début des années 2000. De même, si les pouvoirs publics accordent au jazz beaucoup moins de crédit (s) qu'à la «musique savante », il reste la mieux dotée des "musiques actuelles » et la seule véritablement admise au sein des institutions publiques de formation musicale. Enfin, sur le plan des pratiques de consommation musicale, le jazz, avec la diversité de ses usages (dansants, cultivés, musique de fond) fut longtemps difficile à appréhender à partir de la bipolarité savant/populaire. Sa position dans l'espace social des goûts musicaux s'est cependant nettement rapprochée de celle des «musiques savantes » au cours des dernières décennies.

7 Il n'est pas nécessaire de consacrer de longs développements à la position des « musiques savantes »: le qualificatif traduit la légitimité culturelle qu'elles incarnent depuis longtemps dans le domaine musical (Bourdieu, 1979; Donnat, 1994, 2009; Coulangeon 2003, 2010; Bennett et al., 2009; et sur le public de ces genres musicaux : Menger, 1986; Patureau, 1991 ; Dorin, 2013). Le mouvement réalisé par le jazz paraît plus énigmatique sur le plan sociologique. Il rappelle que les propriétés distinctives des genres musicaux (comme de toute forme d'expression artistique) ne leur sont pas consubstantielles ni attachées de façon permanente mais qu'elles dépendent de la structure changeante de l'ensemble du champ musical. La légitimation du jazz procède de plusieurs processus interdépendants qui sont aussi les principaux indicateurs de sa consécration sociale: l'esthétisation et la classicisation croissante du répertoire (Roueff, 2013), sa reconnaissance institutionnelle et le double mouvement de vieillissement et d'élitisation de son public.

Bien que les données sur les années 1950 demeurent parcellaires, il semble que la position du jazz dans l'espace des styles de vie correspondait plutôt à celle d'un art moyen, masculin et «juvénile». Dans les années 1960, le nombre de spectateurs qui a augmenté durant les deux décennies précédentes connaît un renversement de tendance lié notamment à la vive concurrence de nouveaux genres musicaux (rock et yéyé) qui ravissent au jazz son public adolescent (Tournès, 1999) et probablement aussi une partie de ses fractions populaires. À mesure que les usages dansants se font plus rares, la diversité sociale relative du public tend à s'amenuiser et le recrutement des amateurs semble se déplacer progressivement vers les étudiants et les jeunes actifs. L'analyse des données issues de la première enquête sur les pratiques culturelles des français (1973) confirme le statut d'«art moyen » associé au jazz dans La Distinction et l'hypothèse 
avancée quelques années plus tard par Jean-Louis Fabiani (1986) selon laquelle cet art en voie de consécration recrute alors prioritairement son public au sein des «classes moyennes $»^{3}$.

9 Étape décisive du processus d'habilitation, la politique publique en faveur du « jazz et des musiques improvisées » initiée en 1982 prend place dans les réorientations qui tendent à substituer à l'objectif de la "démocratisation culturelle " celui de la «démocratie culturelle " (Dubois, 1999). Privilégié parmi les genres musicaux qui bénéficient de cette entreprise de décloisonnement ${ }^{4}$, le jazz fait l'objet de plusieurs mesures dont la plus significative en ce sens est sans doute la création en 1986 de l'Orchestre National de Jazz, qui fait du jazz le seul genre à disposer d'un tel ensemble institutionnel en dehors des "musiques savantes". Mais la distribution des crédits s'avère très inégale entre les différentes tendances esthétiques du jazz: c'est principalement son avant-garde, les «musiques improvisées européennes", que vient consacrer le soutien financier à la production, à la création et aux lieux de diffusion. Cette politique renforce les clivages internes qui opposent, d'un côté, le jazz traditionnel et le jazz « moderne » et, de l'autre, les musiciens «novateurs » dont les moins « subversifs » formeront progressivement une avant-garde consacrée, désignée par certains professionnels par la catégorie «jazz contemporain » (Roueff, 2013). Le privilège accordé à ce pôle stylistique, qui correspond le mieux aux valeurs et aux codes esthétiques des formes contemporaines des arts légitimes (danse, théâtre, musique, art contemporains), a sans doute contribué à attirer les catégories les plus diplômées vers le jazz, ne serait-ce qu'en raison de la forte présence de ce style de jazz au sein des programmations des institutions publiques de diffusion du spectacle vivant.

10 Enfin, la reconnaissance du jazz par les pouvoirs publics se traduit par les mesures engagées en faveur de son enseignement dans les conservatoires et les écoles de musique, des plus modestes aux plus prestigieux (les CNSMD de Paris et de Lyon) sans oublier certains départements de musicologie à l'Université5. Avec l'essor simultané des écoles privées, la scolarisation de la transmission du jazz comme facteur de sa légitimation déploie ses effets à partir des années 1980 et contribue, par ailleurs, à l'expansion considérable de l'effectif des jazzmen français.

11 Simultanément à ce mouvement d'institutionnalisation, le public du jazz connaît deux transformations majeures qui vont contribuer à légitimer le genre. La première réside dans le vieillissement du public ( $29 \%$ de retraités en 2008) dont on peut estimer qu'il favorise la respectabilité du jazz. La seconde transformation, l'élitisation du public, est sans doute plus décisive. Entre 1981 et 2008, l'évolution de la distribution par PCS du chef de ménage met en évidence la place croissante occupée par les cadres et professions intellectuelles supérieures, au détriment, principalement, des professions intermédiaires et des ouvriers. La progression des cadres se manifeste de façon plus accentuée au sein du public du jazz que dans la population française, pour les enquêtés qui citent le jazz parmi les genres les plus écoutés et surtout pour la sortie en concert. Un autre indicateur, celui de la répartition du public selon le dernier diplôme obtenu, pointe des évolutions analogues et congruentes avec celles qu'a connues la population française âgée de 15 et plus entre 1989 et 2008 : chute de la part des non diplômés, baisse du nombre d'enquêtés dont le dernier diplôme est le baccalauréat et hausse considérable des diplômés du supérieur. Il s'ensuit que les diplômés du supérieur sont fortement surreprésentés parmi les jazzophiles: en 2008, ils sont presque deux fois plus nombreux qu'au sein de la population française parmi les enquêtés qui écoutent le plus souvent du jazz et plus de 
deux fois au sein du public des concerts. Cette tendance traduit le renouvellement du public par de nouvelles générations de diplômés du supérieur qui se tournent vers le jazz notamment au moment de l'entrée dans la vie active.

Alors que dans les années 1950, la position du jazz dans l'espace des styles de vie correspondait plutôt à celle d'un art moyen, masculin et "juvénile», les indicateurs étudiés montrent son inscription progressive au sein de la culture légitime. Le jazz jouit-il pour autant d'un statut social équivalent à celui des « musiques savantes »? Quelle est sa place dans l'espace plus vaste des goûts musicaux?

\section{Jazz et « musiques savantes » au prisme de la structure sociale de leur public}

13 Pour répondre à ces questions, il convient d'examiner la composition sociale de l'audience des «musiques savantes » et du jazz en les comparant au sein d'un ensemble contrasté de genres musicaux (graphique 1). On procédera ensuite selon le même principe à propos de la structure sociale des publics des concerts au sein d'un ensemble diversifié de sorties culturelles (graphique 2).

\section{Note méthodologique}

Les deux graphiques suivants sont issus de l'exploitation secondaire de l'Enquête sur les pratiques culturelles des Français de 2008 (DEPS, ministère de la Culture). Dans le graphique 1, la structure sociale de l'audience des genres musicaux est appréhendée en croisant la catégorie socioprofessionnelle de l'enquêté avec la variable des « genres musicaux écoutés le plus souvent ». Dans le graphique 2, la structure sociale du public des genres musicaux est appréhendée en croisant la catégorie socioprofessionnelle de l'enquêté avec une série de sorties effectuées au cours des douze derniers mois, parmi lesquelles les concerts des genres musicaux considérés. Si l'âge et le sexe exercent leur influence sur les goûts, ce ne sont pas des variables indispensables pour traiter de la hiérarchie de légitimité culturelle. On a toutefois noté que la disparition du caractère juvénile du goût pour le jazz avait sans doute contribué à sa respectabilité sociale.

Nous avons préféré recourir à une procédure aisément reproductible, celle de l'analyse bi-variée, à partir de l'indicateur simple et bien connu des PCS - qui intègre des dimensions comme le niveau de qualification - plutôt qu'à l'analyse multivariée (sous forme d'ACM ou de CAH, par exemple) qui ne nous parait pas indispensable pour les besoins de la démonstration. Le choix de cet outil offre une bonne représentation de la diversité sociale d'un public.

En raison de la forte présence des retraités au sein de l'audience des genres musicaux étudiés (respectivement $29 \%$ pour le jazz, $43 \%$ pour la musique classique, $50 \%$ pour l'opéra contre $26,5 \%$ pour l'ensemble de la population), la variable utilisée est celle qui indique la PCS des retraités selon leur ancienne profession. Un premier recodage a permis de rapporter les « autres inactifs » et les « élèves et étudiants » à la PCS de leur chef de famille lorsque ce dernier était actif (grande majorité des cas). Un second recodage a été réalisé pour remplacer les 74 « élèves ou étudiants » chefs de famille restants par la PCS de leur père. Nous avons enfin supprimé les 91 
derniers inactifs (sur un échantillon de 5004) qu'il était impossible de rapporter à une catégorie socioprofessionnelle d'actif.

Parmi les genres musicaux retenus dans le graphique 1, les « musiques savantes » sont représentées par « l'opéra » et la « musique classique » (l'effectif de la « musique contemporaine » est trop faible et la signification de cette catégorie trop polysémique pour l'intégrer à l'analyse). Hormis le jazz, le « pop, rock », les « chansons et variétés françaises » et le « rap » ont également été retenus à des fins de comparaison au sein d'un ensemble contrasté de genres musicaux. On retrouve ces genres musicaux dans le graphique 2, hormis le « rap » qui ne figure pas dans la question sur les concerts. La sortie au « spectacle de music-hall, de variétés » a été retenue ici à défaut de sortie qui corresponde mieux au genre « chansons, variétés françaises ». Enfin, la sortie au théâtre offre un bon point de comparaison avec les concerts des genres étudiés dans la mesure où elle est souvent considérée comme la sortie cultivée par excellence.

En raison des transformations de la structure sociale au cours des cinquante dernières années, la prise en considération des retraités selon leur catégorie socioprofessionnelle tend, par rapport à une mesure ne tenant compte que des actifs, à minorer le poids des cadres et professions intellectuelles supérieures, des professions intermédiaires et des employés au profit des autres catégories. En outre, il faut noter que l'enquête Pratiques culturelles des Français sous-représente, par son mode d'échantillonnage, les amateurs réguliers et assidus chez qui les traits caractéristiques du public se trouvent accentués ${ }^{6}$. Malgré ces paramètres qui atténuent leur caractère élitaire, les audiences du jazz, de l'opéra et de la musique classique (graphique 1) possèdent une structure sociale commune qui les distingue de celles des autres genres musicaux : tandis que la catégorie modale y est celle des cadres et professions intellectuelles supérieures, celle des professions intermédiaires est la plus représentée au sein du public du rock, celle des employés pour les chansons et variétés françaises (en lien avec le caractère féminin de ce groupe socioprofessionnel et de ce goût) et celle des ouvriers pour le rap (même remarque du côté masculin). 
Figure 1 : Distribution par groupes socioprofessionnels (inactifs inclus) de l'audience des genres musicaux (" écoutés le plus souvent ») et de la population française

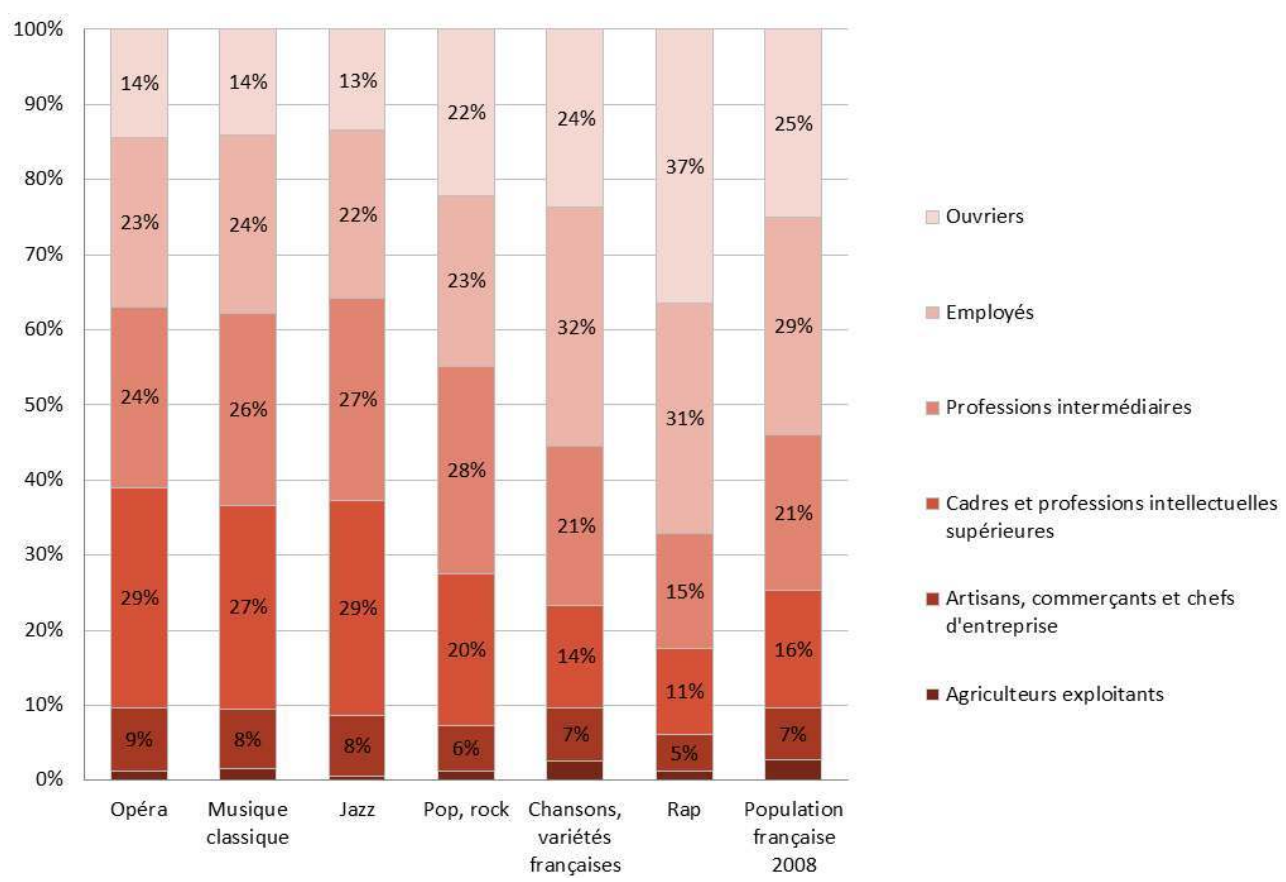

CHAMP : PERSONNES VIVANT EN FRANCE, ÂgÉES DE 15 ANS ET PLUS, ACTIVES OU INACTIVES (RETRAITÉS SELON LEUR ANCIENNE PROFESSION, AUTRES INACTIFS - ÉTUDIANTS, LYCÉENS, PERSONNES AU FOYER - SELON LA PROFESSION DU CHEF DE FAMILLE OU, À DÉFAUT, LA PROFESSION DU PÈRE) ( $\mathbf{N}:$ 4851).

SOURCE : ENQUÊTE SUR LES PRATIQUES CULTURELLES DES FRANÇAIS, DEPS, MINISTÈRE DE LA CULTURE, 2008.

Pour appréhender la hiérarchie sociale des goûts, il importe non seulement de comparer entre elles les structures par PCS de l'audience des genres musicaux mais aussi de les rapporter à celle de la population française. Sous cet angle, bien que le mode d'analyse mis en œuvre soit moins favorable à la thèse des homologies structurales que la lecture en taux d'incidence (Donnat, 2009) ou l'analyse des correspondances multiples qui engendre presque toujours des écarts et une polarisation, une interprétation des résultats selon la tripartition des goûts proposée par Bourdieu (1979) ne semble pas inappropriée. Ainsi, dans une logique relationnelle, on peut voir dans le rap un goût populaire (forte surreprésentation des ouvriers et sous-représentation des professions intermédiaires et des cadres), dans les « chansons et variétés françaises » et le « pop, rock » un goût moyen (écarts relativement faibles avec la population française même si le rock, dont l'audience se distribue de façon analogue à celle du jazz dans les années 1970, apparaît en voie de légitimation) et dans l'ensemble formé par l'opéra, la musique classique et le jazz, le goût musical cultivé ou légitime (nette sous-représentation des classes populaires et forte surreprésentation des cadres). On peut ainsi interpréter ces résultats comme le produit des homologies structurales, à condition de rappeler que ces dernières n'organisent pas des correspondances exclusives entre des catégories de biens culturels et des catégories sociales de consommateurs mais simplement des relations privilégiées entre les unes et les autres (entre l'opéra et les cadres, par exemple, sans pour autant que les ouvriers soient totalement absents du public de l'opéra). 
Le même constat se dégage de manière nettement accentuée dans le cas de la composition sociale des publics des concerts (fréquentés au moins une fois au cours des douze derniers mois), cette dernière pratique étant, comme les sorties culturelles en général, plus sélective socialement que l'écoute d'enregistrements. Ainsi, les cadres et les professions intermédiaires sont plus ou moins surreprésentés dans toutes les sorties retenues.

Figure 2 : Distribution par groupes socioprofessionnels (inactifs inclus) de la population française et de six types de pratiques culturelles

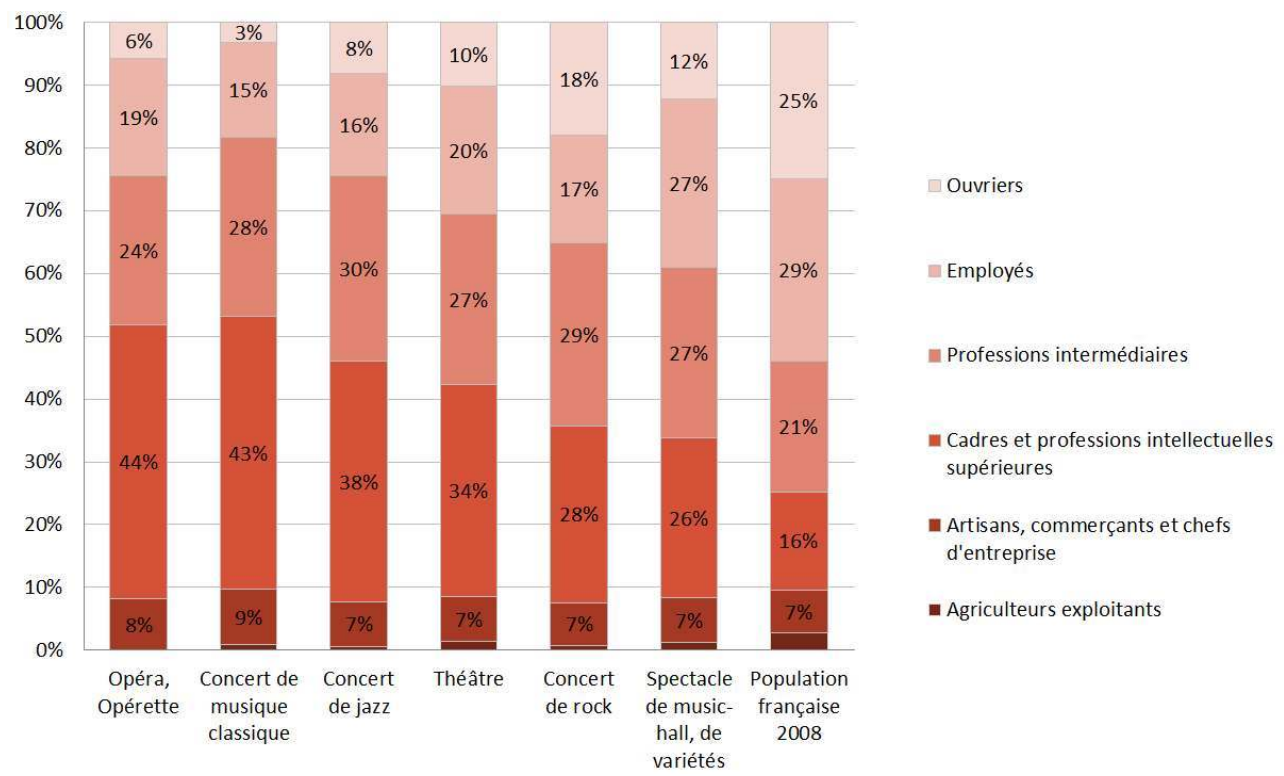

Champ : personnes vivant en France, âgées de 15 ans et plus, actives ou inactives (retraités selon leur ancienne profession, autres inactifs - étudiants, lycéens, personnes au foyer - selon la profession du chef de famille ou, à défaut, la profession du père) ( $N$ : 4851).

Source : Enquête sur les pratiques culturelles des Français, DEPS, Ministère de la Culture, 2008.

Le concert de jazz, se montre désormais plus sélectif socialement que la sortie typiquement cultivée - au théâtre, alors qu'ils se situaient sur le même plan dans les résultats de l'Enquête sur les pratiques culturelles de 1997. Le public des concerts de jazz s'est encore rapproché sous cet angle de ceux de l' "opéra, opérette » et du concert de musique classique. Si le concert de jazz, qui recrute davantage que les autres genres au sein des professions intermédiaires (30\%), demeure un peu moins sélectif socialement que l'« opéra, opérette » et le concert de musique classique (le poids des cadres y demeure légèrement supérieur - respectivement $44 \%$ et $43 \%$ contre $38 \%$ pour le public du jazz), ces trois sorties forment un ensemble relativement homogène qui peut être vu comme la manifestation du goût légitime en matière musicale.

La théorie de la légitimité culturelle a soulevé un certain nombre de questions pertinentes comme celles du degré d'hégémonie de la culture légitime et de l'autonomie relative des cultures populaires (Grignon \& Passeron, 1989), ou encore celle de la commensurabilité des pratiques selon une échelle unidimensionnelle. Bien que les « musiques savantes » et le jazz aient peu à voir avec les cultures populaires ou juvéniles à propos desquelles se sont développées ces interrogations critiques, ces dernières invitent néanmoins à la prudence et, selon le niveau d'observation, à la contextualisation de l'analyse en termes de légitimité culturelle ${ }^{7}$. Toutefois, au niveau macrosociologique auquel la présente analyse se situe, si l'on voit, avec la légitimation du jazz se recomposer 
la sphère de la légitimité culturelle, les résultats invitent à s'inscrire en faux contre la thèse de la disparition de la hiérarchie culturelle au profit d'un archipel de goûts distincts déconnectés des catégories sociales d'appartenance et de la dimension symbolique des rapports (Glévarec, 2013). Cette thèse néglige également le fait que la légitimité culturelle d'un genre musical n'est pas seulement fondée sur la part des classes supérieures au sein de son audience. Elle repose également sur son degré de reconnaissance par l'État, sa place au sein des institutions d'enseignement générales et spécialisées et des instances de consécration (médias culturels, récompenses, académies, manifestations, etc.). Parmi ces dernières, la critique culturelle joue un rôle important que nous allons maintenant tenter d'appréhender à travers le cas récent de la critique en ligne.

\section{Figures d'amateurs et sentiment de légitimité dans les magazines spécialisés}

Dans la presse comme sur Internet, la critique culturelle constitue un terrain peu étudié sous l'angle de la légitimité des formes d'expression artistique. Pourtant, le développement d'une critique a pu être interprété comme le signe d'un accroissement de légitimité de formes culturelles émergentes comme la bande-dessinée (Boltanski, 1975), le cinéma (Darré, 2006) ou le jazz (Fabiani, 1986). Au-delà de leur fonction de gatekeepers (Hirsch, 1972) et de prescripteurs de choix culturels (Schrum, 1991), les critiques interviennent effectivement dans la formation de la valeur des genres artistiques dont ils commentent et évaluent les productions (Allen \& Lincoln, 2004). Au travers des ressources linguistiques et du registre de discours qu'elle mobilise (savant/profane, technique/didactique, expert/amateur, etc.), selon l'univers de références plus ou moins cultivé au sein duquel elle situe les artistes et leurs œuvres, et en fonction de son propre capital symbolique (musicien reconnu vs anonyme, par exemple), la critique est un acteur de la légitimation symbolique (ou, dans certains cas, de la dé-légitimation) du genre ou de la discipline qu'elle prend pour objet. Le souci de légitimité apparaît d'ailleurs dans certains cas au centre de ses préoccupations. Olivier Roueff observe ainsi que, depuis les années 1930, les critiques et promoteurs du jazz ont eu tendance à problématiser la vie du jazz en termes de légitimation. La moindre place accordée depuis les années 1990 à la revendication de la dignité artistique du jazz (hormis lorsqu'il s'agit du différentiel des aides accordées aux institutions du jazz et de la musique classique) peut s'interpréter, à l'inverse, comme un indice de la légitimation acquise du genre (Roueff, 2003).

L'attention portée à la critique comme instance de production de la valeur symbolique des genres musicaux parait d'autant plus justifiée dans le cas des « musiques savantes » et du jazz que, d'une part, ces genres se situent globalement au pôle légitime et relativement autonome (à l'égard des logiques économiques) du champ musical, au sein duquel la critique tient une place plus importante qu'au sein du pôle commercial (Bourdieu, 1992; Shrum, 1991) et que, d'autre part, ils touchent des publics particulièrement diplômés, qui sont parmi les plus enclins à la consommation de discours d'accompagnement.

Cet intérêt pour la critique s'inscrit plus généralement dans l'analyse des médiations sociales qui organisent concrètement la relation entre production et réception des biens symboliques, notamment dans l'étude du rôle des intermédiaires dans la production des valeurs (Lizé et al., 2014) et dans les correspondances qui s'établissent entre l'espace de production et l'espace de consommation sous l'effet des homologies structurales 
(Bourdieu, 1977 ; Lizé, 2010 ; Roueff, 2013), dans lesquelles la légitimité culturelle trouve son principal fondement. Ainsi, à partir d'une enquête lexicographique sur la presse en ligne spécialisée dans le domaine du jazz et dans celui de la musique classique, dont les pratiques s'inscrivent dans la continuité de la critique papier, on se demandera si le discours critique favorise les correspondances que fait apparaître l'analyse statistique entre les genres musicaux étudiés et certains groupes sociaux en les invitant à s'identifier à des figures d'amateurs plus ou moins distinctives.

Les figures de l'amateur qu'Antoine Hennion, Sophie Maisonneuve et Emilie Gomart (2000) ont tenté de cerner par l'enquête, existent également sous la forme de figures prescrites par la critique. Elles désignent des postures à l'égard des biens culturels, des modalités du goût aux caractéristiques suffisamment saillantes et récurrentes pour se voir désignées, nommées, identifiées par l'intermédiaire de catégories qui se définissent dans leurs relations mutuelles au sein de l'espace des goûts pour la musique: «consommateur occasionnel ", « néophyte», « amateur ", «collectionneur », «fan », « initié ", «passionné », « connaisseur ", «érudit », «mélomane », etc. Chacune de ces figures est associée aux significations et à la valeur sociale des modalités du goût qu'elle désigne, en lien avec les principes de catégorisation propres à chaque univers culturel. Comment sont désignées les personnes qui aiment le jazz par comparaison à celles qui aiment la musique classique dans la critique spécialisée sur Internet? Quelles significations sont associées à chaque catégorie employée pour nommer l'amateur? La légitimité des pratiques s'ancre-t-elle dans les mots employés par les critiques et les journalistes pour désigner les amateurs de ces deux genres musicaux?

Le journalisme culturel, de même que les amateurs passionnés, s'est emparé d'Internet comme outil de diffusion. Il existe ainsi plusieurs sites consacrés au jazz et à la musique classique. Parmi eux, deux webzines, citizenjazz.com et resmusica.com (consacré aux " musiques savantes ») forment le terrain textuel de cette recherche. Apparus au début de la décennie 2000, ces deux sites ont été choisis parce qu'ils sont, en langue française, les plus importants dans leur domaine respectif par le nombre d'articles en ligne et la fréquence de publication (voir l'encadré ci-dessous). Ils ont également été sélectionnés parce qu'ils traitent de façon relativement œcuménique des styles subsumés respectivement sous les catégories jazz et «musique savante » et que leur contenu est similaire à ceux de leurs homologues papier : chacun d'eux présente un grand nombre de chroniques de disques, de livres et de DVD et en récompensent certains («élu » par citizenjazz.com, les "clés ResMusica »). Ils proposent également des entretiens avec des artistes, des dossiers, l'agenda et de nombreux comptes-rendus de concerts, le tout assorti de multiples photographies. Ce qui les distingue notamment des magazines de presse réside dans le nombre important de comptes-rendus de concerts et dans la moindre présence de publicités - qui s'accompagne potentiellement d'une plus grande indépendance à l'égard des principaux annonceurs issus de l'industrie du disque et du spectacle (voir Béra, 2003). Un dictionnaire des compositeurs est en outre hébergé sur le site resmusica.com. Si le volume de ce dernier site dépasse celui de citizenjazz.com, tous deux mettent chaque mois en ligne l'équivalent d'un magazine de presse (resmusica.com se présente même comme un quotidien : ainsi, en 2006, 1312 articles ont été mis en ligne ${ }^{8}$ ). Ils disposent de rédacteurs relativement nombreux dont certains écrivent également dans la presse spécialisée : «Plus de 50 rédacteurs répartis dans sept pays, dont les grands pays francophones que sont la France, la Belgique, la Suisse, le Canada élaborent le contenu de ResMusica.com. Pour établir la ligne rédactionnelle [...] et gérer le site, une 
dizaine de collaborateurs assurent la cohésion nécessaire au développement de ce projet. $»^{9} \mathrm{Au}$ sein de l'équipe rédactionnelle de citizenjazz.com et de ses collaborateurs occasionnels figurent ou ont figuré des personnes qui exercent le métier, soit de musicien (ne), soit de journaliste musical, soit de photographe, soit qui travaillent dans le milieu musical (certains ont un diplôme de 3ème cycle en musicologie) ${ }^{10}$. Bien qu'ils publient sur Internet, il s'agit donc moins de « critiques profanes » que de «critiques experts " pour reprendre la catégorisation de Marc Verboord (2010) en lien avec le développement, via Internet, de l'exercice de la critique par des amateurs. Le contenu éditorial est ici contrôlé et ces sites se réfèrent aux standards du journalisme culturel des magazines et revues spécialisées. Par ailleurs, les chiffres de fréquentation dont ils font état indiquent qu'ils ont de nombreux lecteurs ${ }^{11}$.

\section{Les webzines spécialisés dans le jazz et les musiques savantes}

Au moment de l'enquête (en 2007), la fermeture récente de jazzvalley.com fait de citizenjazz.com le principal site de jazz en France. D’apparition récente, les sites jazzbreak.com, culturejazz.fr et le blog Les dernières nouvelles du jazz, eux aussi dédiés à l'actualité du jazz dans sa diversité (éditorial, chroniques de disques, de livres, de DVD et de concerts, interviews, articles, etc.), sont moins alimentés que citizenjazz.com et jazzbreak.com est plus centré sur le Sud de la France. Site créé en 2001, bananierbleu.fr propose à peu près les mêmes rubriques mais se consacre au seul « jazz caribéen ». Il en va de même pour djangostation.com, créé en 2004 et spécialisé dans le jazz manouche. Depuis l'enquête, jazzbreak.com a disparu et plusieurs magazines de la presse spécialisée ou revues plus confidentielles ont créé ou développé leur site en mettant en ligne plus de contenu : Jazz Hot diffuse l'essentiel de son contenu gratuitement sur Internet, Jazz Magazine (après fusion avec Jazzman en 2009) alimente uniquement son site de comptes-rendus de concerts et de festivals et Improjazz (consacré à l'avant-garde) est passé en ligne. Citizenjazz.com demeure toutefois le principal webzine consacré au jazz.

Les sites consacrés à la musique classique sont un peu plus nombreux. La Scena musicale, site de la revue québécoise du même nom, publie depuis la fin des années 1990 des actualités, des articles, des chroniques, un forum et des liens. Le nombre d'articles demeure toutefois moins important que sur resmusica.com, de même que pour altamusica.com et anaclase.com, créés respectivement en 1999 et en 2003, qui sont les sites qui s'en rapprochent le plus par leur contenu : brèves et chroniques, critiques de disques et de concerts, dossiers, nombreux entretiens (chefs d'orchestre, musiciens, etc.), etc. Mis en ligne en 2000, concertclassic.com se consacre en particulier à l'actualité des concerts, avec une billetterie en ligne, même s'il dispose aussi d'une rubrique « journal » (news, chroniques, comptes-rendus). Créé en 2000, Travers-sons est un magazine en ligne et une base de données discographiques conçus par la Médiathèque de la Communauté francophone de Belgique ; il propose éditorial, actualités, critiques de $\mathrm{CD}$, dossiers thématiques, livres, notices d'opéras et biographies de compositeurs. La publication d'articles est cependant irrégulière (elle semble d'ailleurs s'être arrêtée en 2010). Apparu dans la première moitié des années 2000, critique-musicale.com et symphozik.info ont été créés par des passionnés, " sans prétention aucune » peut-on lire sur le second ; ils ne se donnent pas pour objectif de suivre l'actualité musicale : le premier propose 4000 critiques d'œuvres, 680 notices sur des compositeurs et des sélections d'œuvres; le second propose des 
ressources diverses : biographies, dossiers, tests d'oreille ou de culture, blagues et jeux musicaux. Enfin, d'autres sites et blogs sont apparus après l'enquête, sans pour autant que resmusica.com ne perde sa place centrale : Drôles de gammes, blog sur le site de Rue 89 (août 2007) ; classiqueinfo.com (novembre 2007) ; classicstoday.com (2009) ; classissima.com (2009) ; tutti-magazine.fr (2009) ; vivre-musique-classique.fr (2010) ; Classique d'aujourd'hui (2011) ; inconcerto.nicematin.com (2011); classiquenews.com (2012) ; crescendo-magazine.be (2012) ; classicagenda.fr (2014) ; nomadmusic.fr (2014).

L'examen de ces magazines diffusés sur Internet et les caractéristiques que nous venons d'indiquer autorisent à penser que les schèmes classificatoires qui président à la désignation des amateurs, à la mise en scène de l'excellence et de la médiocrité en chaque domaine, ne diffèrent pas significativement de ceux des revues spécialisées diffusées sur support papier. Ce sont bien les structures symboliques de l'univers du jazz, d'un côté, et de l'univers de la musique classique, de l'autre, ainsi que le degré de légitimité culturelle de ces deux genres musicaux qu'il semble ainsi possible d'approcher.

\section{Procédure de recherche sur Internet}

Cet encadré présente la procédure mise en œuvre au début du mois de mai 2007 (moins d'un an avant l'enquête sur les pratiques culturelles des Français sur laquelle s'appuie la partie précédente) pour rechercher les termes qui désignent « les personnes qui aiment le jazz » sur le site citizenjazz.com et « les personnes qui aiment la musique classique » sur le site resmusica.com.

Nous avons utilisé le mode « recherche avancée » de Google qui permet de concentrer la recherche de mots sur un site déterminé. Nous avons ainsi recherché sur les deux sites tous les termes susceptibles de désigner les amateurs. Pour restreindre le relevé des citations aux discours des journalistes ou des critiques, les forums de discussion des lecteurs ont été exclus de l'investigation. Nous avons pris soin d'exclure les doublons liés aux pages de présentation des articles, aux pages « imprimer », etc. Une procédure de vérification a été mise en œuvre pour citizenjazz.com avec le moteur de recherche Yahoo. Elle a livré moins de résultats dans l'ensemble, mais le recoupement a fait apparaître une vingtaine de résultats supplémentaires (sur plus de 300), tous termes confondus, sans modifier la structure d'ensemble de la distribution.

Pour concentrer la recherche sur des figures d'amateurs et non retenir tous les mots susceptibles de les qualifier, les termes recherchés l'ont été sous forme de substantifs : les emplois comme adjectif ou comme participe passé de certains termes ( « passionné » par exemple) ont été exclus du recensement, de même que l'emploi du terme « amateur » lorsqu'il désigne les musiciens non professionnels. Le singulier, le pluriel et le féminin de chacun des termes retenus ont été soumis à l'investigation et inclus dans le dénombrement. Ils ne sont signalés dans le tableau présenté plus bas que lorsqu'ils apparaissaient dans le corpus.

Les termes « auditeur », « spectateur » et " public » ont également été exclus de la recherche. Ils reviennent évidemment souvent et, comme les autres termes, interpellent le lecteur et le mettent en scène d'une certaine façon. Mais ils réfèrent 
trop strictement à une situation, celle de l'écoute, de l'assistance au concert. De plus, ils sont relativement neutres dans la mesure où ils ne qualifient pas le rapport à la musique « des personnes qui aiment », comme le font les autres termes, mais celui des personnes qui écoutent à un moment et dans un lieu donnés. Les retenir transformerait la distribution sans qu'on puisse en tirer une analyse pertinente.

Le mot « spécialiste(s) » n'a pas été exclu de la recherche mais du décompte final qui apparaît dans le tableau, parce qu'il ne désigne pas seulement une personne qui aime le jazz mais le plus souvent aussi un professionnel de cet univers (il faudrait, sinon, inclure aussi les musiciens).

Le tableau suivant présente la fréquence des différents termes employés pour désigner les « personnes qui aiment le jazz » au sein du corpus citizenjazz.com et celles « qui aiment la musique classique » en ce qui concerne resmusica.com.

Tableau 1 : Nombre d'occurrences des termes désignant les « personnes qui aiment le jazz » et les "personnes qui aiment la musique classique"

\begin{tabular}{|c|c|c|c|c|}
\hline $\begin{array}{l}\text { Termes désignant les } \\
\text { personnes qui aiment... }\end{array}$ & $\begin{array}{l}\text {..le jazz } \\
\text { (citizenjazz.com) }\end{array}$ & Rang & $\begin{array}{l}\text {...la musique classique } \\
\text { (resmusica.com) }\end{array}$ & Rang \\
\hline Amateur(s) & 140 & 1 & 214 & 2 \\
\hline $\operatorname{Fan}(\mathrm{s})^{12}$ & 47 & 2 & 55 & 5 \\
\hline Admirateur(trice)(s) & 26 & 3 & 75 & 3 \\
\hline Passionné(e)(s) & 24 & 4 & 46 & 6 \\
\hline Néophyte(s) & 19 & 5 & 44 & 7 \\
\hline Mélomane(s) & 19 & 6 & 461 & 1 \\
\hline Connaisseur(s) & 13 & 7 & 66 & 4 \\
\hline Collectionneur(s) & 9 & 8 & 22 & 10 \\
\hline Amoureux(euse) & 9 & 9 & 44 & 8 \\
\hline Jazzophile(s) & 8 & 10 & 0 & 17 \\
\hline Aficionado(s) & 8 & 11 & 26 & 9 \\
\hline (Non-)initié(e)(s) & 6 & 12 & 20 & 11 \\
\hline Adeptes & 4 & 13 & 13 & 13 \\
\hline Novice(s) & 3 & 14 & 10 & 14 \\
\hline $\operatorname{Mordu}(\mathrm{e})(\mathrm{s})$ & 3 & 15 & 5 & 15 \\
\hline Discophile(s) & 0 & 16 & 17 & 12 \\
\hline
\end{tabular}




\begin{tabular}{|l|l|l|l|l|}
\hline Audiophile(s) & 0 & 17 & 5 & 16 \\
\hline Total & 338 & & 1123 & \\
\hline
\end{tabular}

Champ : ensemble des pages des sites citizenjazz.com et resmusica.com, en mai 2007, hormis les forums de discussion des lecteurs, les pages de présentation des articles et les pages « imprimer » pour écarter les doublons.

Source : citizenjazz.com (consulté 03.05.2007) et resmusica.com (consulté 05.05.2007).

Ce recensement montre tout d'abord que le même ensemble de termes est employé pour désigner les amateurs de jazz et les amateurs de musique classique. Toutefois, les termes les plus utilisés, ceux d'" amateur», de "mélomane» et de "fan», le sont dans des proportions significativement différentes dans chaque genre musical. Le volume du corpus resmusica.com étant beaucoup plus important que celui de citizenjazz.com, ce ne sont pas tant les nombres d'occurrences en valeur absolue qui sont significatifs que leur valeur relative. Le graphique suivant permet de les visualiser et de comparer la place relative qu'occupe chacun des termes au sein des deux corpus.

Figure 3 : Termes désignant les « personnes qui aiment le jazz » et les « personnes qui aiment la musique classique »

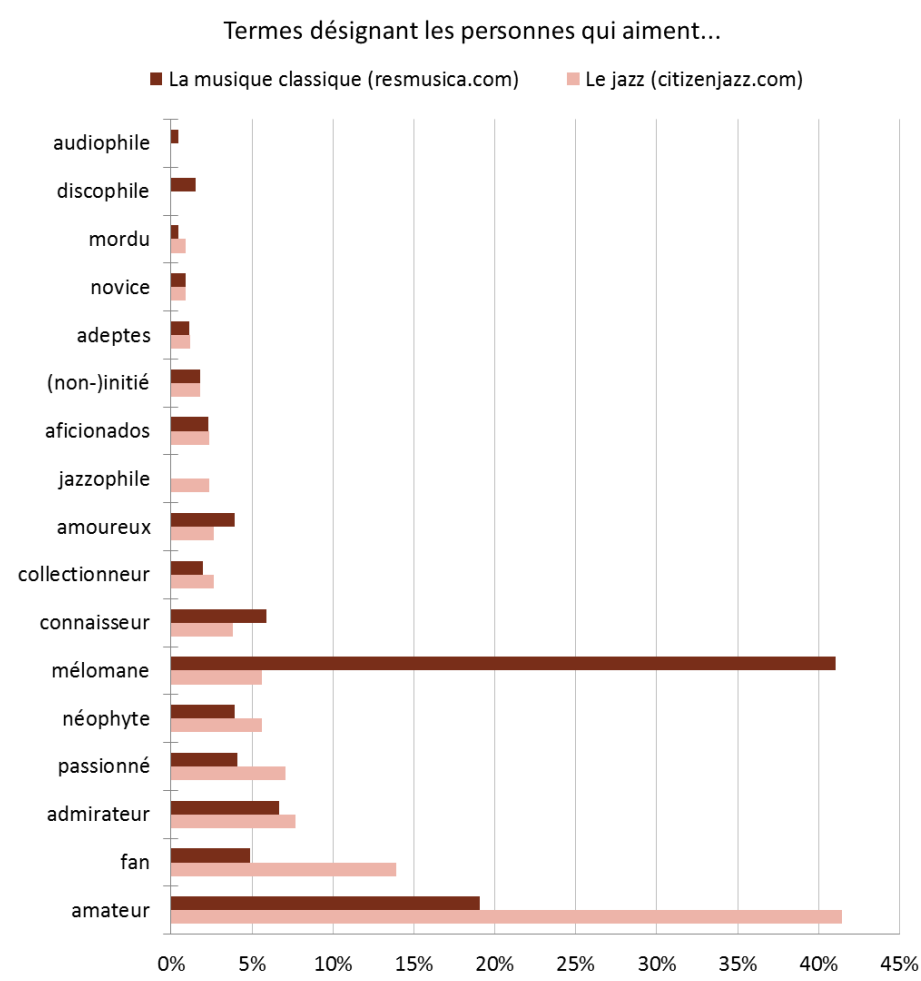

Champ : ensemble des pages des sites citizenjazz.com et resmusica.com, en mai 2007, hormis les forums de discussion des lecteurs, les pages de présentation des articles et les pages « imprimer » pour écarter les doublons.

Source : citizenjazz.com (consulté 03.05.2007) et resmusica.com (consulté 05.05.2007).

27 Le graphique fait clairement apparaître la prédominance pour chaque genre d'un terme qui rassemble la même proportion d'occurrences : alors qu' « amateur ${ }^{13}$ » apparaît comme le vocable privilégié ( $41 \%)$ par les commentateurs spécialisés pour désigner ceux qui aiment le jazz, les personnes qui aiment les musiques savantes sont le plus souvent 
désignées par le terme de "mélomane ${ }^{14} »(40 \%)$. Ces derniers se voient dans un second temps désignés par le terme " amateurs » (19\%), si bien que « mélomane » et " amateur » représentent $61 \%$ du total des occurrences pour les musiques savantes. Les «fans » ne sont pas totalement absents mais ne viennent qu'en cinquième position avec seulement $5 \%$ des occurrences.

En revanche, lorsqu'ils ne sont pas des "amateurs", ceux qui aiment le jazz sont prioritairement des «fans" (13\%) ou des «admirateurs» ( $8 \%)$, deux termes au sens proche ${ }^{15}$, alors qu'ils ne sont désignés par le vocable «mélomane » qu'en 6ème position ( $6 \%$ des cas). Cette faible présence de "mélomane» pourrait témoigner du rapport ambigu des professionnels et des amateurs de jazz à la " musique savante » : si le goût du jazz s'est construit en partie par opposition à celui de la musique classique, celle-ci a souvent servi sous la plume des jazzologues de point de comparaison visant à légitimer le jazz (Roueff, 2013). On notera enfin que la figure du "connaisseur", qui dénote une maitrise de l'objet de goût par le savoir, vient en quatrième position pour le classique (6 \% ) alors qu'elle n'apparaît qu'au septième rang pour le jazz (la relation s'inversant pour le terme « néophyte »).

Il faut revenir brièvement sur la catégorie «fan » qui occupe la seconde position dans le corpus de citizenjazz.com et qui, avec celle d'admirateur (8\%), regroupe $21 \%$ des occurrences. Mot anglais, abréviation de fanatic dont l'usage s'est répandu en France à partir de la Libération, "fan" désigne une passion ou une admiration excessive pour quelqu'un ou quelque chose ${ }^{16}$. Généralement connoté de façon péjorative, il désigne une conduite parfois perçue comme « irrationnelle » qui, le plus souvent associée à la culture juvénile ou à la culture populaire, s'oppose au détachement distingué de l'esthète. Même si, comme le note Philippe Le Guern, «la notion de "fan" reste floue et à géométrie variable et peine à caractériser un univers diversifié par ses membres (fans de foot, de séries TV, de groupes de rock...) et ses pratiques (tous les fans ne participent pas à des fans-clubs) »(Le Guern, 2006 : 373), elle regroupe des pratiques qui ont en commun d'être exclues de la sphère des arts consacrés. L'examen des usages de la notion de «fan » et des pratiques qu'elle désigne doit toutefois se prémunir des dérives du nominalisme et du substantialisme. Dans la presse comme dans les échanges quotidiens, le terme «fan » est parfois employé de façon métaphorique pour désigner un intérêt marqué pour un artiste ou une passion qui n'empruntent pas nécessairement les traits typiques du fan. Qu'en estil plus précisément de l'usage du mot au sein de nos deux corpus ? Le terme « fan(s) » ne renvoie pas systématiquement à des styles mineurs, ni nécessairement à des comportements culturellement « suspects » :

Sorti au mois de juin dernier, le tout nouveau "Trio Wanderer» était attendu comme un grand événement dans le milieu des fans de la formation mais aussi par les adorateurs de la musique de chambre de Brahms. (Chronique de CD sur resmusica.com. URL : www.resmusica.com/2006/10/11/brahms-le-wanderer/) Les fans de Griffin ou de Grossman apprécieront sûrement, mais aussi ceux qui aiment un jazz simple et sans complexes. (Chronique de CD sur citizenjazz.com. URL : www.citizenjazz.com/Johnny-Griffin-Steve-Grossman.html)

Pourtant, le terme «fan » est celui qui est privilégié lorsqu'il s'agit de stigmatiser le comportement en concert ou l'absence de discernement de certains amateurs :

Car Bruno Berenguer a fourni un travail sérieux, documenté et complet, évitant tout autant l'hagiographie de fan énamouré (il émet par exemple des réserves sur les capacités vocales de Denise Duval à incarner Manon) que les effets people les 
plus déplacés. (À propos d'un livre sur resmusica.com. URL : www.resmusica.com/2004/03/31/denise-duval-par-bruno-berenguer/)

Le pari était fou: Magma en résidence quatre semaines d'affilée au Triton, la salle de concerts des Lilas! A raison d'une capacité maximale d'environ 160 personnes, cela revenait à remplir le Zénith... Pas gagné d'avance, même si les fans de Magma, on le sait, sont "cumulards" de nature : on soupçonne même certains irrécupérables d'avoir été présents aux vingt concerts! (À propos de concerts sur citizenjazz.com. URL : www.citizenjazz.com/Magma-Mythes-et-Legendes-I.html)

31 En outre, le mot «fan » est associé à l'admiration des vedettes, c'est-à-dire, dans les deux univers, des artistes qui connaissent la consécration en partie au moins selon des critères médiatiques ou commerciaux.

C'est que, vraie diva, elle a tout d'une star, pas seulement pour magazines sur papier glacé : voix de lait et de miel, présence rayonnante et simple pourtant, disponible, souriante pour la horde de ses fans lors de l'obligatoire et interminable séance de signature. (À propos d'un récital de Renée Fleming sur resmusica.com. URL : www.resmusica.com/2005/11/23/le-chant-star/)

Enfin, s'il est parfois employé comme métaphore qui souligne simplement l'intensité d'un goût, sa connotation péjorative demeure bien présente dans nombre de cas, alors qu'elle est très rare s'agissant des autres termes qui désignent « ceux qui aiment » le jazz ou la musique classique. Ainsi, au-delà des occurrences disséminées dans les deux revues en ligne, l'emploi du mot «fan(s) » apparaît au cœur d'articles qui mettent en scène avec condescendance la passion «excessive », la dévotion «irrationnelle», les postures culturellement « indignes » $d^{\prime}$ ' amoureux transis » qui font figure de contre-modèles de l'amateur ou du mélomane distingué ${ }^{17}$.

Ces répertoires de postures disponibles à l'égard de la musique semblent s'organiser autour de formes d'excellence différentes au sein du monde de la musique classique et au sein du monde du jazz. On peut considérer que la catégorie la plus fréquente « mélomane » pour la musique classique, « amateur » pour le jazz - renvoie au goût défini comme légitime, du moins par la critique, définition qui s'appuie aussi sur le mépris ou la condescendance pour des modalités discréditées de la passion telles que vu précédemment. Sur les deux sites, la valeur symbolique des figures d' "amateur » et de " mélomane » est souvent soulignée par les adjectifs "grand », " averti ", " raffiné », " connaisseur", "éclairé ", autant de qualificatifs qui ne sont jamais employés avec "fan", comme si cela devait donner lieu à des oxymores. Les extraits suivants témoignent de la dignité culturelle conférée à ces figures, en particulier à celle du mélomane :

...le Gros Cube du saxophoniste Alban Darche constitue un de ces exemples de collectif apprécié des "amateurs éclairés", de la presse dite "spécialisée" mais quasiinconnu du public. (URL: www.citizenjazz.com/Le-Gros-Cube-Lpt-a-lErmitage.html)

On est heureux de « retrouver » les standards de l'avant-guerre, même si l'amateur averti risque d'être surpris par des arrangements à contrecourant... (URL : www.citizenjazz.com/Las-Vegas-Rhapsody.html)

...les mélomanes dignes de ce nom n'oublieront jamais son interprétation désormais légendaire des œuvres pour piano et violon de Béla Bartók avec André Gertler. (URL : www.resmusica.com/2005/10/28/joseph-jongen-un-grand-musicien-belgemagnifiquement-rehabilite/)

Réduire la distance qui sépare le néophyte du mélomane averti, tel était l'objectif premier $\mathrm{du}$ producteur Daniel Toscan $\mathrm{du}$ Plantier... (URL: www.resmusica.com/2006/09/27/don-giovanni-de-joseph-losey-editionrestauree-2006/) 
Ce monde de la sensibilité et de l'émotion est encore dévolu aux plus mélomanes

d'entre nous. (URL : www.resmusica.com/2004/06/05/le-temps-des-vocalise/) comparaison initiale en considérant que les catégories employées pour désigner celles et ceux qui aiment un genre musical sont constitutives de la valeur sociale de ce genre et de ses admirateurs. Les modes de désignation participent en effet d'un système de classements qui traduit la valeur sociale et artistique conférée à chaque genre et aux goûts afférents. La fréquence du terme mélomane semble consubstantielle de la légitimité traditionnelle du goût pour les musiques savantes et de celle des formes musicales regroupées sous cette catégorie. Si le terme " amateur ", privilégié pour désigner ceux qui aiment le jazz, est rarement connoté de façon péjorative, il reste relativement neutre dans la mesure où il est employé pour désigner la relation à de nombreux objets de prédilection dont l'ensemble excède le domaine artistique. En revanche, "mélomane " caractérise en propre l'amour de la musique. Or, au regard des résultats, l'amour de la musique semble réservé aux personnes qui écoutent les musiques savantes tandis que les amateurs d'autres genres musicaux ne paraissent pas dignes du vocable «mélomane ». Sous la plume des rédacteurs de citizenjazz.com, les occurrences de ce terme ne désignent jamais de façon explicite les amateurs de jazz, comme s'il y avait une contradiction entre "mélomane» et "jazz». Dans le même temps, le monde des musiques savantes a tendance à s'accaparer le terme «musique »: dans la presse et les ouvrages spécialisés, lorsqu'il s'agit de parler de musique en toute généralité, ce seul terme désigne assez souvent, sans complément ni qualificatif, les musiques savantes et exclut alors implicitement les autres formes musicales ${ }^{18}$. Le nom même du webzine «resmusica» témoigne de cette monopolisation symbolique de la musique: implicitement, le «fait musical » serait tout entier contenu dans les formes musicales dont traite ce site. La formule latine « resmusica » marque également l'enracinement de la musique classique dans la culture européenne des humanités, bénéficiant de la noblesse qui est encore socialement attribuée à cette dernière, alors que "citizenjazz » renvoie à la culture états-unienne d'où le jazz est issu.

\section{Conclusion}

Médiations qui instaurent un lien spécifique entre sujet et objet du goût, catégories discursives qui renvoient à des catégories distinctes d'action, de pensée, de comportement qu'il convient d'adopter à l'égard du jazz ou de la musique classique, les figures d'amateurs prescrites par la critique doivent simultanément être appréhendées sous l'angle de leur valeur symbolique comme des médiations entre le champ de production des œuvres et l'espace socialement hiérarchisé des consommateurs. L'analyse du rôle de ces médiations et de la place des intermédiaires que sont les critiques dans les relations d'homologie structurale qui s'établissent entre chaque genre musical et la position sociale de ses consommateurs (voir la 1ère partie) nécessiteraient une analyse plus large du discours critique et de ses producteurs.

On voudrait toutefois conclure sur l'enjeu de cet article : le prolongement de l'analyse de la légitimité culturelle des «musiques savantes » et du jazz par l'investigation conduite sur la critique en ligne. Sous cet angle, il convient d'interroger l'inégale valorisation du goût qui se dégage de l'examen des figures d'amateur prescrites par la critique Internet (2ème partie) au regard des indices de la légitimité culturelle de chacun des genres 
musicaux étudiés (1ère partie). Au premier abord, on remarque que le différentiel de dignité culturelle entre les deux goûts musicaux qui se dégage de la comparaison des termes employés pour désigner l'amateur ne correspond pas tout à fait à la forte proximité qui prévaut dans la distribution sociale des deux publics. Toutefois, il faut rappeler, à cet égard, que l'enquête sur les pratiques culturelles des Français de 2008 est la première à partir de laquelle la composition sociale de l'audience du jazz («genres écoutés le plus souvent») rejoint aussi parfaitement celle des "musiques savantes", tandis que celle du public des concerts de jazz demeure, on l'a vu, légèrement moins élevée socialement que celle des concerts de musique classique. De plus, à l'ancienneté de l'accès des genres à la légitimité culturelle correspond l'ancienneté de l'appartenance des auditeurs aux classes dominantes. En effet, à partir de la même enquête, Philippe Coulangeon (2010) montre que l'effet du diplôme prime pour l'écoute du jazz alors que c'est l'effet de l'origine sociale qui l'emporte pour l'écoute de la musique classique ${ }^{19}$. Le processus de consécration du jazz a certes déplacé les frontières de la légitimité culturelle en matière musicale, mais ces variations conséquentes selon l'origine sociale montrent l'attachement des "héritiers " à la musique classique, autrement dit la socialisation familiale au sein des classes dominantes joue un rôle plus important dans l'intérêt pour les formes canoniques de la légitimité musicale. Il est tentant de mettre en relation l'ancienneté de l'appartenance du public des musiques savantes à la classe dominante et la posture aristocratique qui consiste, dans le discours critique, à réserver à ces formes musicales l'exclusivité du terme "musique » et de la figure du mélomane. À l'aune des figures d'amateurs prescrites par la critique spécialisée, l'ennoblissement culturel et social des genres artistiques est manifestement soumis à l'épreuve du temps, perceptible notamment dans l'inertie des catégories discursives. On peut sans doute voir, en effet, dans l'inégale valeur symbolique de chacun des genres l'effet de l'ancienneté de l'accès à la légitimité culturelle, qui place les "musiques savantes» du côté de la légitimité traditionnelle et le jazz au pôle des genres artistiques récemment promus. L'inertie des catégories discursives tend ainsi peut-être davantage que l'évolution sociologique du public de chaque genre à préserver ses quartiers de noblesse culturelle à la «musique savante ».

37 Ces variations selon l'origine sociale et les écarts quant au sentiment de légitimité culturelle que les critiques en ligne du jazz et des « musiques savantes » renvoient de leur genre d'appartenance à leurs lecteurs rejoignent par ailleurs le fossé qui sépare la place accordée à ces deux formes d'expression musicales dans les aides allouées par l'État. Si le jazz se voit privilégié au sein des «musiques actuelles », les fonds réservés à la « musique savante » représentent toujours l'essentiel (environ $90 \%$ ) des sommes allouées à la musique par les pouvoirs publics. De même, les équipements qui leurs sont principalement dédiés (tout récemment : la Philharmonie de Paris) ainsi que leur place dans les institutions d'enseignement, ou encore dans les médias de la culture cultivée (France Culture, France Musique, Arte, Télérama) traduisent bien la dignité et l'autorité symbolique qui leur sont conférées par les élites politiques et culturelles qui, «à défaut d'imposer la conformité dans les pratiques effectives, sont en mesure d'imposer la norme de la légitimité culturelle, d'agir sur le jeu et sur la formation des profits symboliques. » (Pinto, 2013 : 93). 


\section{BIBLIOGRAPHIE}

ALLEN Michael Patrick \& LINCOLn Anne E. (2004). «Critical discourse and the cultural consecration of American films », Social Forces, 82 (3), pp. 871-894.

ATKINSON Will (2011). «The context and genesis of musical tastes: Omnivorousness debunked, Bourdieu buttressed », Poetics, 39 (3), pp.169-186.

BENNETT Tony, SAVAge Mike, silva Elizabeth, WARDE Alan, GAYo-CAL Modesto, wriGHT David (2009). Culture, Class, Distinction, London, Routledge.

BÉRA Matthieu (2003). «Critique d'art et/ou promotion culturelle? », Réseaux, 21 (117), pp. 153-187.

BOLTANSKI Luc (1975). « La constitution du champ de la bande dessinée », Actes de la recherche en sciences sociales, 1 (1), pp. 37-59.

BOURDIEU Pierre (1977). « La production de la croyance : contribution à une économie des biens symboliques ", Actes de la recherche en sciences sociales, 13 (1), pp. 3-43.

BOURDIEU Pierre (1979). La Distinction. Critique sociale du jugement, Paris, Minuit. BOURDIEU Pierre (1992). Les Règles de l'art. Genèse et structure du champ littéraire, Paris, Seuil. COULANGEON Philippe (1999). Les Musiciens de jazz en France, Paris, L'Harmattan. COULANGEON Philippe (2003). « La stratification sociale des goûts musicaux. Le modèle de la légitimité culturelle en question ", Revue française de sociologie, 44(1), pp. 3-33.

COULANGEON Philippe (2004). Les Musiciens interprètes en France. Portrait d'une profession, Paris, La Documentation Française.

COULANGEON Philippe (2010). « Les métamorphoses de la légitimité », Actes de la recherche en sciences sociales, 181-182, pp. 88-105.

CURIEN Nicolas \& MOREAU François (2006). L'industrie du disque, Paris, La Découverte.

DARRÉ Yann (2006). « Esquisse d'une sociologie du cinéma », Actes de la recherche en sciences sociales, 161-162, pp. 122-136.

DMDTS (2001). «Les musiques actuelles dans les établissements d'enseignement spécialisé contrôlés par l'état », Mesure pour Mesure, 9 [en ligne]. URL : www.culturecommunication.gouv.fr/content/download/98427/880494/version/1/ file/2001_musiques_etablissement.pdf (consulté le 09.05.2016). DONNAT Olivier (1994). Les Français face à la culture. De l'exclusion à l'éclectisme, Paris, La Découverte. Donnat Olivier (2009). Les Pratiques culturelles des Français à l'ère numérique. Enquête 2008, Paris, La découverte/Ministère de la Culture et de la Communication.

DORIN Stéphane (2013). « Dissonance et consonance dans l'amour de la musique contemporaine. Les limites de l'omnivorisme musical dans l'auditoire de l'Ensemble intercontemporain ", in Coulangeon Philippe \& Duval Julien (dir.) Trente ans après La Distinction de Pierre Bourdieu, Paris, La Découverte, pp. 99-112. 
DUBoIs Vincent (1999). La Politique culturelle. Genèse d'une catégorie d'intervention publique, Paris, Belin.

DUBOIS Vincent, MÉON Jean-Matthieu \& PIERRU Emmanuel (2009). Les Mondes de l'harmonie. Enquête sur une pratique musicale amateur, La Dispute, Paris.

FABIANI Jean-Louis (1999). « Carrières improvisées : théories et pratiques de la musique de jazz en France », in Moulin Raymonde (dir.) Sociologie de l'art, Paris, L'Harmattan [1986], pp. 231-245.

FRANÇOIS Pierre (2008). Les Critiques d'art contemporain. Une perspective de sociologie économique, Paris, Ministère de la Culture, Délégation aux arts plastiques.

GLÉVAREC Hervé (2013). La Culture à l'ère de la diversité, La Tour d'Aigues, Éd. L'Aube.

GRIGNON Claude \& PASSERON Jean-Claude (1989). Le Savant et le populaire. Misérabilisme et populisme en sociologie, Paris, Le Seuil/Gallimard.

HENNION Antoine, MAISONNEUVE Sophie \& GOMART Émilie (2000). Figures de l'amateur. Formes, objets pratiques de l'amour de la musique aujourd'hui, Paris, La Documentation Française.

HIRSCH Paul M. (1972). « Processing fads and fashions: An organization-set analysis of cultural industry systems », American Journal of Sociology, 77 (4), pp. 639-659.

KING Timothy (2007). « Does film criticism affect box office earnings? Evidence from movies released in the US in 2003 », Journal of Cultural Economics, 31(3), pp. 171-186.

LE GUERN Philippe (2006). «De l'amour de l'art ou du... : les fans, un objet problématique pour la sociologie de la culture et des médias ? », in Deniot Joëlle \& Pessin Alain (dir.) Les Peuples de l'art, Tome 2, Paris, L'Harmattan, pp. 365-383.

LIZÉ Wenceslas (2008). L'Amour du jazz : sociologie du goût musical, Thèse de doctorat, Paris, Université Paris 8 .

LIzÉ Wenceslas (2010). « Le goût jazzistique en son champ. L'espace parisien de la jazzophilie », Actes de la recherche en sciences sociales, 181-182, pp. 60-87.

LIZÉ Wenceslas \& ROUEFF Olivier (2010). Étude sur les publics et les non-publics du jazz en Bourgogne, commanditée par le Centre Régional du Jazz en Bourgogne, avec le soutien du DEPS du ministère de la Culture.

LIZÉ Wenceslas, NAUDIER Delphine \& SOFIO Séverine (dir.) (2014). Les Stratèges de la notoriété. Intermédiaires et consécration dans les univers artistiques, Paris, Éditions des archives contemporaines.

MENGER Pierre-Michel (1986). «L'oreille spéculative. Consommation et perception de la musique contemporaine ", Revue française de sociologie, 27 (3), pp. 445-479.

PASQUieR Dominique, BEAUDoIn Valérie \& LEGON Thomas (2014). « Moi je lui donne 5/5 ». Paradoxes de la critique amateur en ligne, Paris, Presses des Mines.

PATUREAU Frédérique (1991). Les pratiquants de l'art lyrique aujourd'hui, Paris, Maison des Sciences de l'Homme.

PERRENOUd Marc (2007). Les Musicos. Enquête sur des musiciens ordinaires, Paris, La Découverte.

PETERSON Richard A. (2005). « Problems in comparative research: the example of omnivorousness ", Poetics, 33 (5/6), pp. 257-282. 
PETERSON Richard \& SIMKUS Albert (1992). « How Musical Tastes Mark Occupational Status Groups » in Lamont Michèle \& Fournier Marcel (dir.) Cultivating Differences. Symbolic Boundaries and the Making of Inequality, Chicago, Londres, The University of Chicago Press, pp.152-186.

PETERSON Richard A. \& KERN Roger (1996). « Changing Highbrow Taste: From Snob to Omnivore », American Sociological Review, 61 (5), pp. 900-907.

PINTO Louis (2013). « Du bon usage de La Distinction », in Coulangeon Philippe \& Duval Julien (dir.) Trente ans après La Distinction de Pierre Bourdieu, Paris, La Découverte, pp. 83-95.

PRIEUR Annick, ROSENLUND Lennart \& SKJOTT-LARSEN Jacob (2008). « Cultural capital today: a case study from Denmark », Poetics, 36 (1), pp. 45-71.

ROBETTE Nicolas \& ROUEFF Olivier (2014). « An eclectic eclecticism: Methodological and theoretical issues in the quantification of cultural omnivorism », Poetics, 47, pp. 23-40.

RouefF Olivier (2003). « De la légitimité du jazz », in Donnat Olivier \& Tolila Paul (dir.) Le(s) Public (s) de la culture, Paris, Presses de Sciences Po, pp. 319-342.

ROUEFF Olivier (2013). Jazz, les échelles du plaisir. Intermédiaires et culture lettrée en France au vingtième siècle, Paris, La Dispute.

SAVAGE Mike \& GAYO Modesto (2011). «Unravelling the omnivore: A field analysis of contemporary music tastes in the United Kingdom », Poetics, 39 (5), pp. 337-357.

sCHMUTZ Vaughn (2005). « Retrospective Cultural Consecration in Popular Music. Rolling Stone's Greatest Albums of All Time », American Behavorial Scientist, 48 (11), pp. 1510-1523.

SHRUM Wesley (1991). «Critics and publics: Cultural mediation in highbrow and popular performing arts », American Journal of Sociology, pp. 347-375.

TOURNÈs Ludovic (1999). New Orleans sur Seine. Histoire du jazz en France, Paris, Fayard.

VAN REES, C. J. (1987). « How reviewers reach consensus on the value of literary works », Poetics, 16 (3/4), pp. 275-294.

VERBOORD Marc (2010). « The legitimacy of book critics in the age of the Internet and omnivorousness. Expert critics, internet critics and peer critics in Flanders and the Netherlands ", European Sociological Review, 26 (6), pp. 623-637.

WARDE Alan, WRIGHT David \& GAYO-CAL Modesto (2007). « Understanding Cultural Omnivorousness: or the myth of the cultural omnivore », Cultural Sociology, 1(2), pp. 143-164.

\section{ANNEXES}

Illustration 1 : Un compte rendu de concert sur le site citizenjazz.com (mars 2004) 


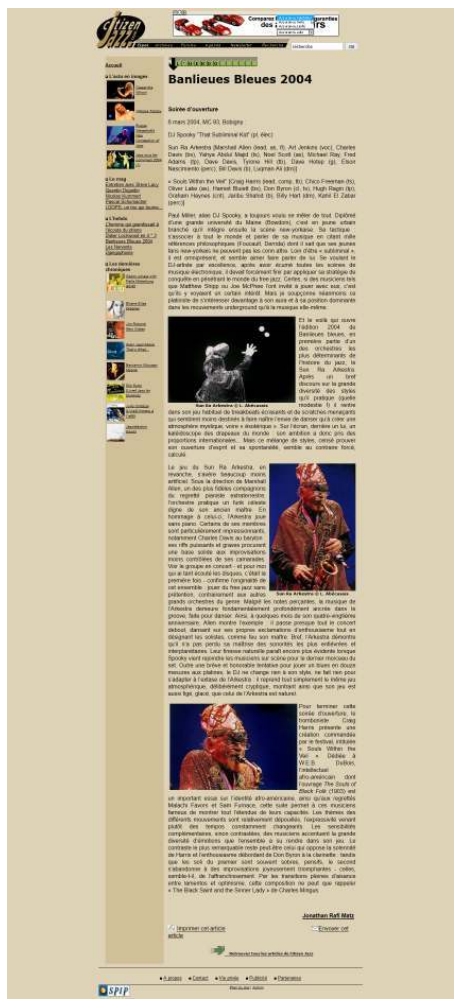

Source : www.citizenjazz.com/Banlieues-Bleues-2004.html (consulté 03.05.2007).

\section{Illustration $2:$ La page d'accueil du site resmusica.com}

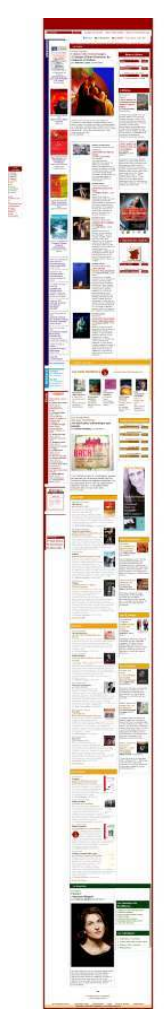

Source : www.resmusica.com (consulté 29.04.2007) 


\section{NOTES}

1. Je remercie Anne-Sophie Béliard et Sidonie Naulin ainsi que les deux rapporteurs anonymes pour leurs remarques et leurs suggestions sur la première version de ce texte.

2. En 2007, la France comptait 32213 artistes professionnels de la musique et du chant contre 11820 en 1982 (source : ministère de la Culture).

3. Le traitement de la question des "genres musicaux écoutés le plus souvent » montre que la CSP la plus représentée pour le jazz est celle des «cadres moyens» suivie par celle des «employés» et la part des «ouvriers» est légèrement plus élevée que celle des «cadres supérieurs » (Lizé, 2008).

4. En progression nettement plus soutenue que celle du budget de la culture lui-même, le montant des sommes allouées au jazz représente en 1995 plus de $45 \%$ du total des crédits alloués à l'ensemble «chanson, jazz, rock» (Coulangeon, 1999: 25). En 2005, selon le Centre d'Information du Jazz, le budget alloué aux « jazz et musiques improvisées » est de plus de quatre millions d'euros dont près d'1,1 million en crédits centraux et 800000 au seul Orchestre National de Jazz. Les trois millions restants sont des crédits déconcentrés affectés aux conservatoires, écoles associatives, résidences et aides aux grands ensembles et collectifs. Ce montant est sans commune mesure avec le poids du jazz dans les pratiques culturelles associées aux autres genres musicaux.

5. Tandis qu'au début des années 1980, il n'existe qu'une classe de jazz dans un Conservatoire National de Région (CNR) et deux en Écoles Nationales de musique (ENM), en 2002, le jazz est enseigné dans 252 établissements publics, soit trois écoles sur quatre qui déclarent offrir un enseignement jazz ou des activités d'ensembles constitués (soit 10000 élèves et plus de 600 professeurs de jazz dans le seul domaine public). (DMDTS, 2001).

6. C'est le constat qui ressort de la comparaison avec les résultats d'enquêtes sur le public d'une pratique ou d'un genre particulier. Ainsi, pour le public du jazz, les proportions d'hommes, de cadres et professions intellectuelles supérieures et de diplômés de l'enseignement supérieur sont plus importantes dans les résultats de l'enquête menée sur les publics des concerts de jazz en Bourgogne (Lizé \& Roueff, 2010) que dans les résultats de l'enquête Pratiques culturelles des Français, sans que les caractéristiques sociodémographiques des habitants de cette région ne soient susceptibles d'expliquer ces écarts.

7. Un exemple d'analyse nuancée et empiriquement fondée nous est donné par Dubois et al. (2009) sur la pratique musicale au sein des orchestres d'harmonie.

8. Dont 702 dans la rubrique «Écouter Voir Lire », 520 dans la rubrique «La Scène » et 67 dans «Le magazine» dont 49 entretiens et portraits (URL: http://www.resmusica.com/ qui_sommes_nous.php3).

9. http://www.resmusica.com/qui_sommes_nous.php3

10. Le co-fondateur et directeur de la publication, Mathieu Jouan, a d'abord été rédacteur en chef du webzine So What (1995-2000) et il est membre de l'Académie du jazz depuis 1999. On peut également citer parmi les rédacteurs : Nicolas Fèvre, Anne Legrand, Philippe Méziat, Philippe Fréchet, Evrim Evci, Extralys, Stanley Péan, Eric Gasnault, Patrick Garbous, Véronique Pernin, Xavier Encinas, Xavier Matthyssens.

11. "ResMusica.com publie des statistiques de fréquentation qui sont labellisés par Weboscope depuis fin 2005. Entre les derniers trimestres de 2005 et 2006, ResMusica aura vu son trafic augmenter de $57 \%$ en visites, soit 90.000 visites faites en décembre 2006 par 70.000 lecteurs ayant consulté près de 200.000 pages vues.» (URL: http://www.resmusica.com/ qui_sommes_nous.php3). Pour citizenjazz.com, « le succès croissant de notre média est confirmé aujourd'hui par l'adhésion volontaire de près de 120000 personnes à notre newsletter et la visite 
mensuelle de $40 \quad 000$ internautes (avril 2007).» (www.citizenjazz.com/ agenda_inscription_v2.html).

12. Les occurrences du terme «fanatique » (5 pour le jazz, 3 pour le classique) ont été regroupées avec celle de «fan ». Pour le jazz, les quatre occurrences du terme « jazzfan » ont également été regroupées sous le mot «fan ».

13. Selon le dictionnaire Trésor de la langue française informatisé, l'amateur est une «personne qui manifeste un goût très vif pour l'art. [...] L'accent est fréquemment mis sur la sûreté intuitive du jugement qui résulte de la fréquentation des œuvres d'art. (Quasi-) synonyme : Connaisseur. [...] À cet emploi valorisateur et laudatif, s'oppose parfois un emploi péjoratif (avec une idée de goût excessif ou peu éclairé), tant au sens général que dans le domaine de l'art ».

14. Selon le dictionnaire Trésor de la langue française informatisé, le mélomane est une "personne qui aime beaucoup la musique ». Aucune connotation péjorative n'est signalée. L'étymologie du terme renvoie à « celui qui est fou de musique ".

15. Selon le dictionnaire Trésor de la langue française informatisé, "admirateur » désigne une " personne qui admire un être, une œuvre ». Il a en commun avec «fan » la " nuance péjorative » qu'il peut revêtir « dans le vocabulaire mondain ».

16. Le Robert donne comme seul synonyme de fan le mot "groupie», plus péjoratif (et sexiste), qui qualifie les ferventes admiratrices des vedettes du rock ou des variétés.

17. Par exemple : www.citizenjazz.com/Les-Nervants.html ou www.resmusica.com/2006/02/06/ divas-la-force-dun-destinet-la-jouissance-de-leau-de-rose/ ou encore www.resmusica.com/2005/07/22/promenade-aux-rives-de-la-folie/

18. Par exemple, l'ouvrage de référence, La Musique du XX $X^{e}$ siècle de Jean-Noël von Der Weid (Fayard, 1992, plusieurs fois réédité) traite essentiellement des musiques savantes, principalement de la musique contemporaine. Seuls des liens sont ponctuellement établis avec le jazz, le rock et les musiques du monde, mais l'ouvrage, malgré son titre, ne traite pas de l'histoire de ces genres. Dans le même sens, la rubrique «Histoire de la musique » des resmusica.com ne traite que des musiques savantes.

19. «La musique classique est ainsi citée par près de la moitié des diplômés de niveau bac +3 issus des classes supérieures ( $48 \%$ ), alors qu'elle ne l'est que par $31 \%$ des diplômés de même niveau lorsqu'ils sont issus des classes populaires, soit moins que les diplômés de niveau bac d'origine supérieure (35\%) et même que les sans-diplôme de même origine supérieure (34 \%). [...] À niveau de diplôme équivalent, les écarts selon l'origine sont de moindre ampleur pour le jazz. La hiérarchie des diplômes semble ici globalement primer : les diplômés de l'enseignement supérieur de niveau bac+2 et bac+3 d'origine populaire citent plus souvent le jazz parmi leurs genres de prédilection que les non-diplômés d'origine supérieure. »(Coulangeon, 2010 : 101)

\section{RÉSUMÉS}

L'enjeu de l'article est de montrer comment une investigation conduite sur les catégories investies par la critique musicale en ligne permet de prolonger l'analyse d'un processus social plus large : celui de la légitimation culturelle. Dans un premier temps, l'étude comparée de la légitimité culturelle des «musiques savantes" et du jazz à partir d'indicateurs classiques, notamment les caractéristiques des publics saisies à travers le traitement des enquêtes sur les pratiques culturelles des Français (de 1973 à 2008), montre que le jazz a connu au cours des cinquante dernières années un processus de légitimation qui le rapproche des «musiques 
savantes » dans l'espace socialement hiérarchisé des goûts. Suivant l'ambition de diversifier les indicateurs en direction notamment des manières de commenter, de fréquenter et d'apprécier les œuvres, on s'intéresse dans un second temps aux différentes façons de désigner l'amateur dans les deux genres musicaux à partir d'une recherche lexicographique sur deux webzines spécialisés, citizenjazz.com et resmusica.com. Les figures d'amateurs privilégiées par la critique en ligne, qui sont autant de postures prescrites à l'égard de la musique, varient suivant les genres et participent ainsi d'un système de classements qui traduit la valeur sociale et artistique conférée à chaque genre et aux goûts afférents.

This article aims to show how an investigation into categories invested by online music critics extends the analysis of a broader social process: that of cultural legitimation. First, the comparative study of cultural legitimacy of "learned music" and jazz based on classic indicators, including the social features of audiences as reflected in surveys on French cultural practices (1973 to 2008), shows that jazz has experienced a process of legitimation over the last fifty years that brings it close to "learned music" in the social hierarchy of tastes. Following the ambition to diversify indicators towards ways to comment, to attend and to enjoy music, I then consider the different ways to designate the music lovers in both musical genres from a lexicographical research on two specialized webzines: citizenjazz.com et resmusica.com. Figures emphasized by online critics, which are all prescribed postures with regard to music, vary among genres and thus contribute to a classification system that reflects the social and artistic value attributed to each genre and related tastes.

\section{INDEX}

Mots-clés : critique, webzine, jazz, musiques savantes, légitimité culturelle, processus de légitimation, figures d'amateurs

Keywords : critics, webzine, jazz, learned music, cultural legitimacy, legitimation process, music lovers figures

\section{AUTEUR}

\section{WENCESLAS LIZÉ}

Université de Poitiers, GRESCO 